\title{
VELOCITY STATISTICS AND SPECTRA IN THREE-STREAM JETS
}

Tobias Ecker

K. Todd Lowe

Wing F. Ng

Virginia Tech

\section{Brenda Henderson}

NASA Glenn Research Center

Stewart Leib

Ohio Aerospace Institute

AIAA SciTech 2016

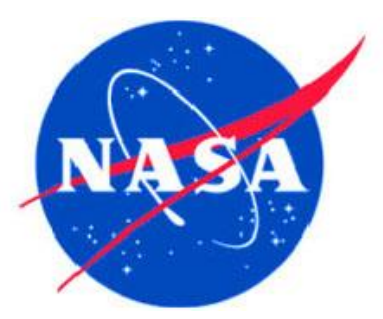




\section{Overview}

- Background

- Time-resolved Doppler Global Velocimetry

- Facility and experiment

- Turbulence results

\section{- Conclusions and next steps}

\section{Acknowledgements:}

This work supported by Commercial Supersonic Technology Project in the Advanced Air Vehicles Program and the US Office of Naval Research (ONR) through the Hot Jet Noise Basic Research Challenge

Mark Wernet for PIV data and support in planning and setup
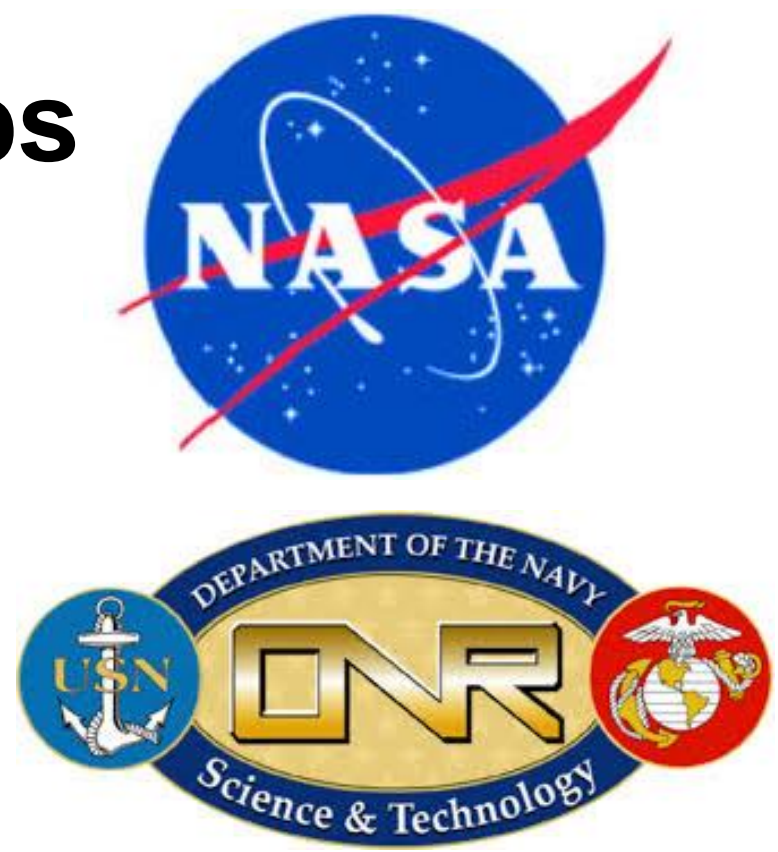


\section{Motivation}

Persistent Jet Noise Challenge

- Supersonic transport unique jet noise problems

- Substantial impact on health of service persons

Instrument development goals

- New tools for turbulent plume details

- Data for development of models for jet noise prediction

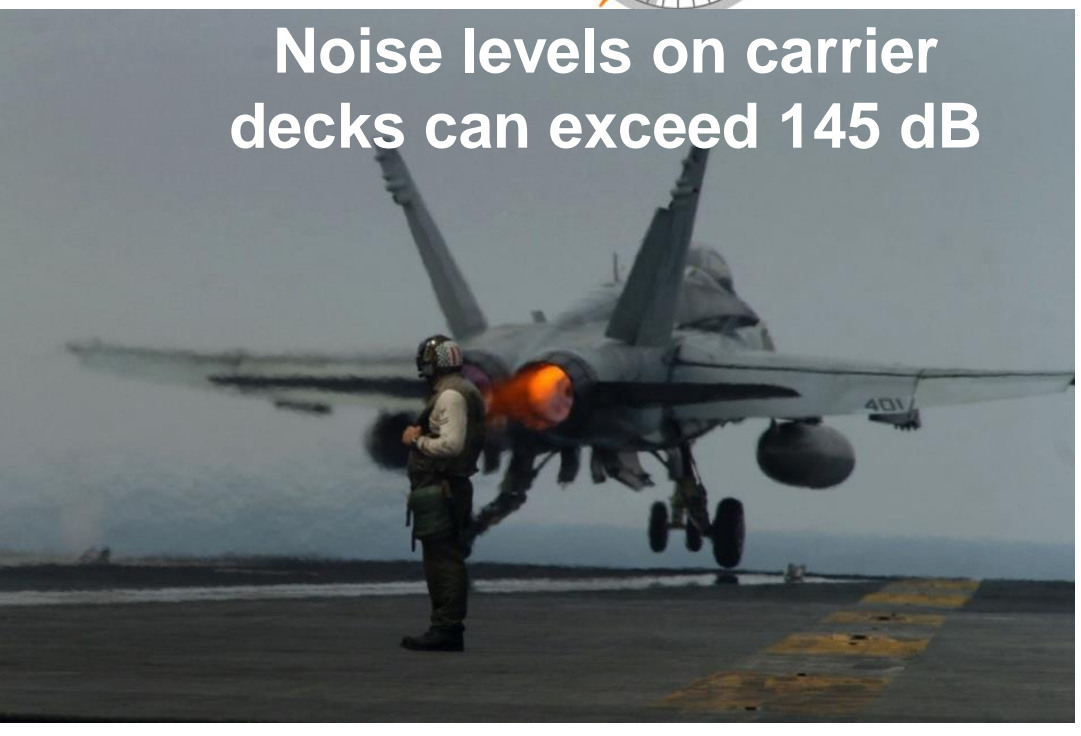

Continuous dB

Permissible Exposure Time

$85 \mathrm{~dB}$

$88 \mathrm{~dB}$

$91 \mathrm{~dB}$

$94 \mathrm{~dB}$

$97 \mathrm{~dB}$

$100 \mathrm{~dB}$

$103 \mathrm{~dB}$

$106 \mathrm{~dB}$

$109 \mathrm{~dB}$

$112 \mathrm{~dB}$

$115 \mathrm{~dB}$

8 Hours

4 hours

2 hours

1 hour

30 minutes

15 minutes

7.5 minutes

3.75 minutes $(<4 \mathrm{~min})$

1.875 minutes $(<2 \mathrm{~min})$

$.9375 \mathrm{~min}(\sim 1 \mathrm{~min})$ $.46875 \min (\sim 30 \mathrm{sec})$ 


\section{Background}

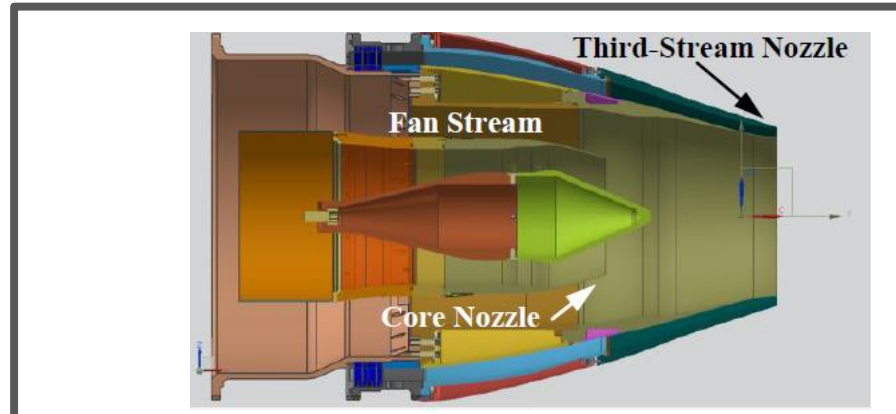

Henderson (2012): Axisymmetric third stream:

- High frequency noise reduction

- Reduced impact with forward flight
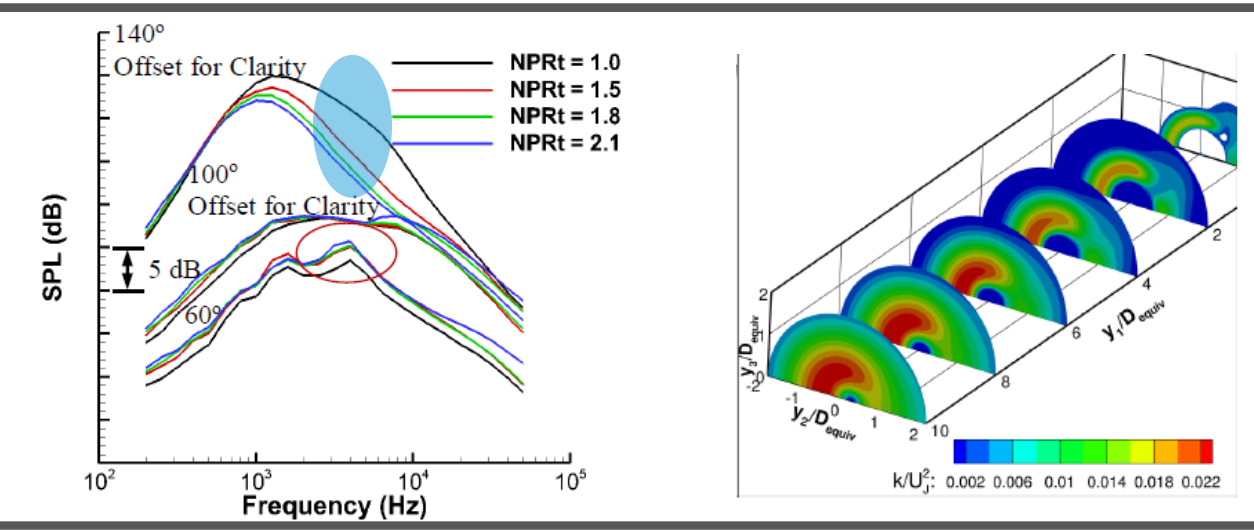

Henderson, Leib and Wernet (2015): Asymmetric third stream

- Dramatic reductions downstream

- Redistribution of TKE

Papamoschou et al. (2016): Even more creative third streams:

- RANS shows redistribution of TKE

- Dramatic noise downstream reduction
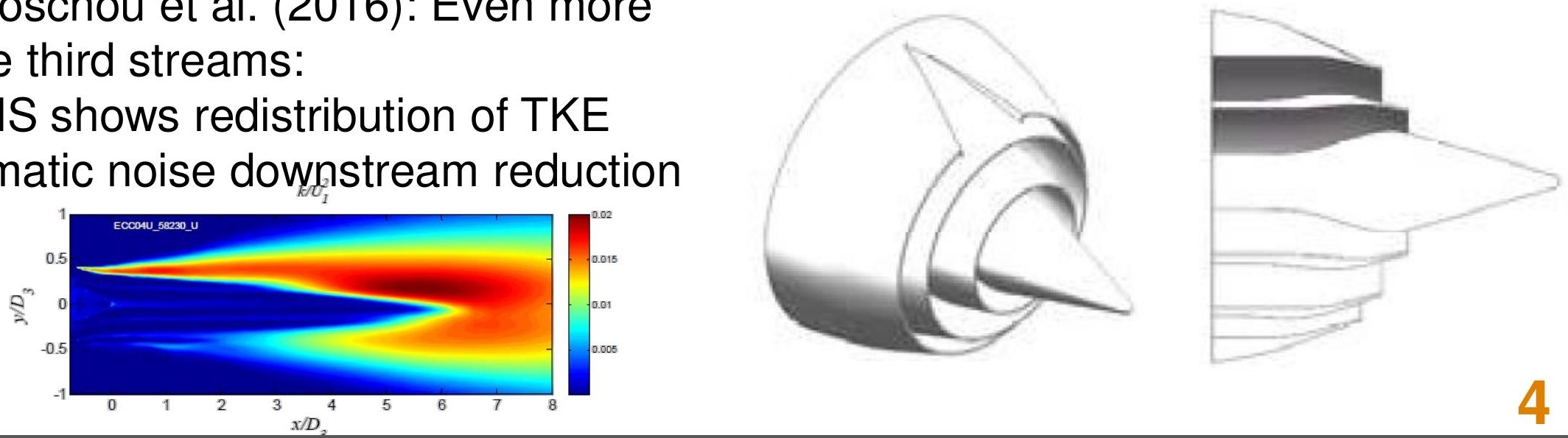


\section{High speed jet instrumentation \&}

\section{measurements}
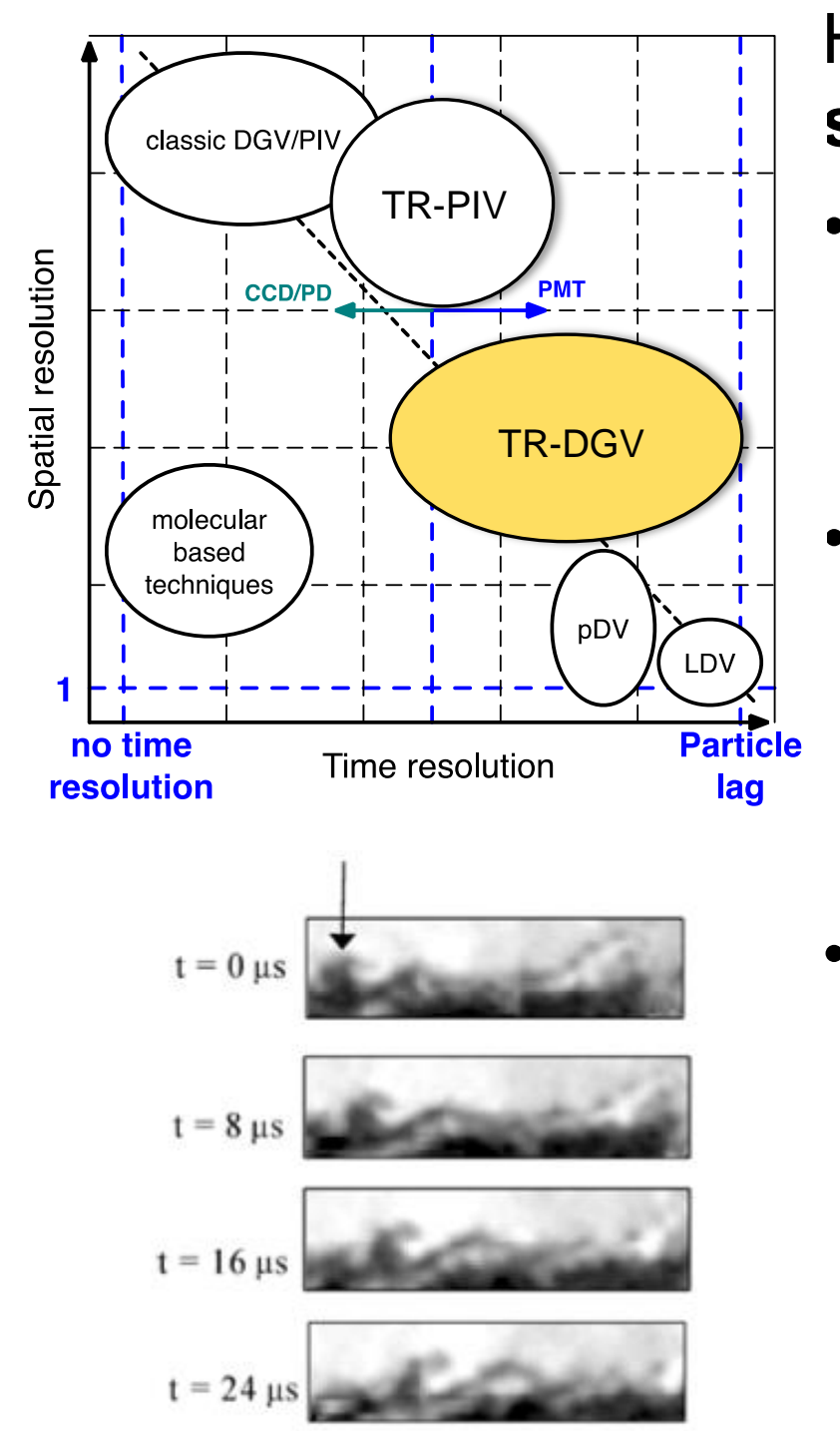

High-data rate, non-intrusive measurements in cold supersonic jets/ hot subsonic jets:

- LDV, Kerhervé et al. (EIF 2004)

- 2-point LDV in cold supersonic jet

- Space-time correlations

- TR-PIV, Wernet and Bridges (2007)

- Up to $50 \mathrm{kHz}$ in subsonic hot jets

- Spectral development

- Space-time correlations

- Megaherz rate DGV (Thurow et al.,2005):

- max. $1 \mathrm{MHz}$ camera sampling rate (presented results up to $250 \mathrm{kHz}$ ), 1-comp

- Supersonic cold jet

- Convective velocities 


\section{Doppler Global Velocimetry}

\section{Basics:}

- First developed by Komine (Northrop Co) in 1990, refined by Meyers and Komine (1991)

- Mie scattered light is sent trough a molecular gas cell (e.g lodine) and its frequency transduced to intensity (24 "optical frequency-to-intensity converter")

- Using a reference or calibration the Doppler frequency shift can be determined:

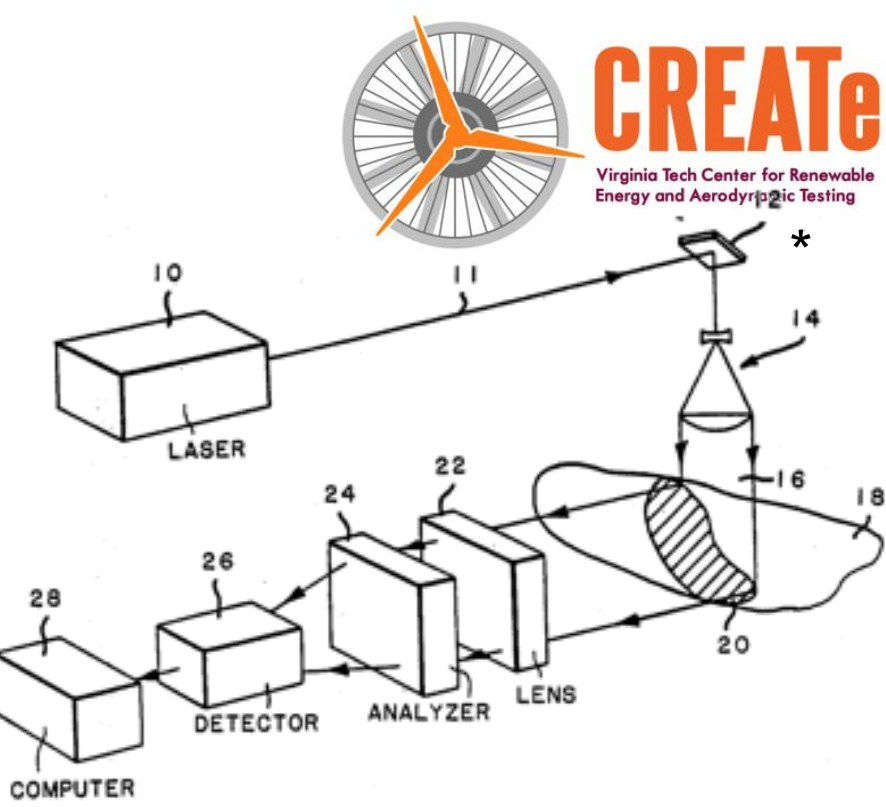

$$
f_{D}=\frac{\vec{o}-\vec{i}}{\lambda} \cdot \vec{V}
$$

- Considered to be optimal for high speed flow due tc absolute error

- Conventional systems have (mean) uncertainties in the range $\pm 0.5 \mathrm{~m} / \mathrm{s}$ to $\pm 3 \mathrm{~m} / \mathrm{s}$

${ }^{*}$ Komine, System for measuring velocity field of fluid flow utilizing a laser-Doppler spectral image converter, US Patent No. 4919 536, 1990

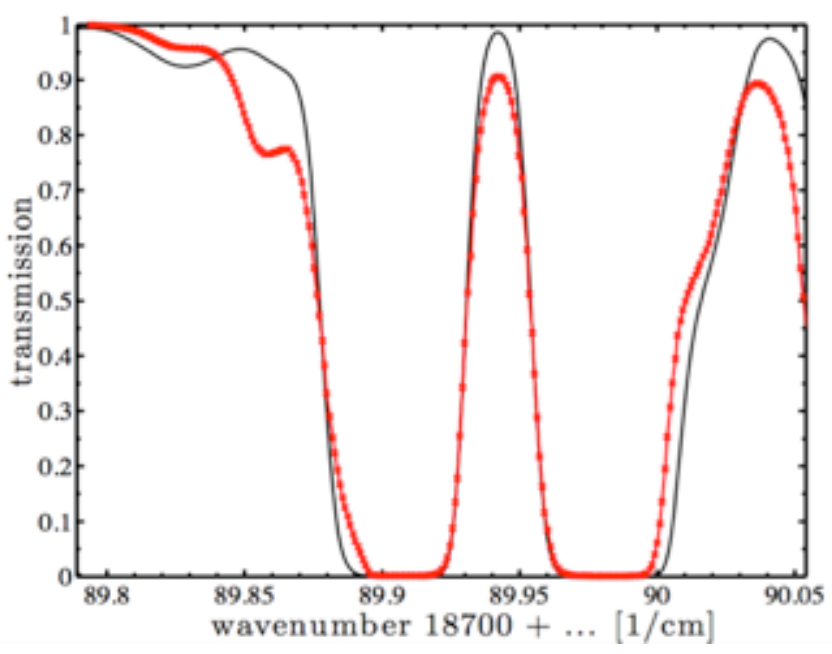

lodine cell transmission scan 


\section{Time-resolved Doppler Global Velocimetry}
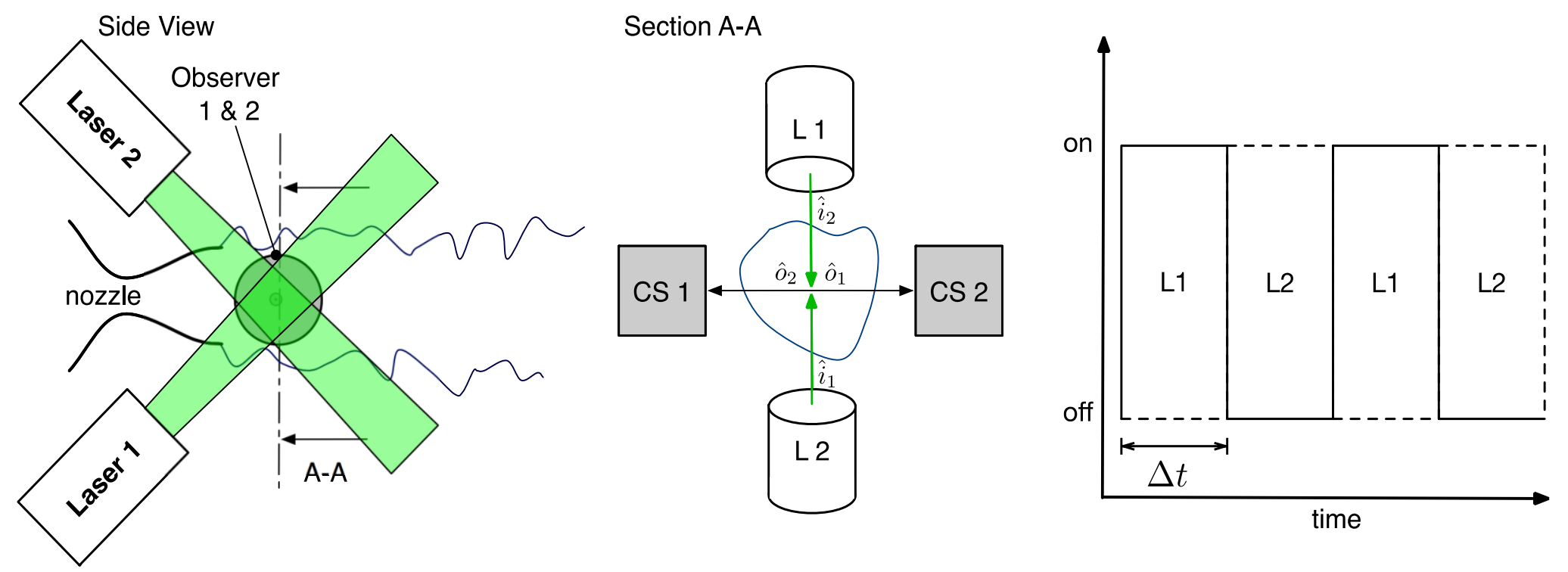

Three-velocity component operation by velocity

$f_{D}=\frac{\vec{o}-\vec{i}}{\lambda} \cdot \vec{V}$ multiplexing:

2 laser beam directions +2 collection directions = 3 linearly independent Doppler directions + 1 redundant measurement 


\section{Previous TR-DGV}

Thurow et al. (2005)

- High-Speed camera based

Ecker et al. (2014)

- Single point 3 component TR-DGV (PMT based)

- First approach to laser beam multiplexing

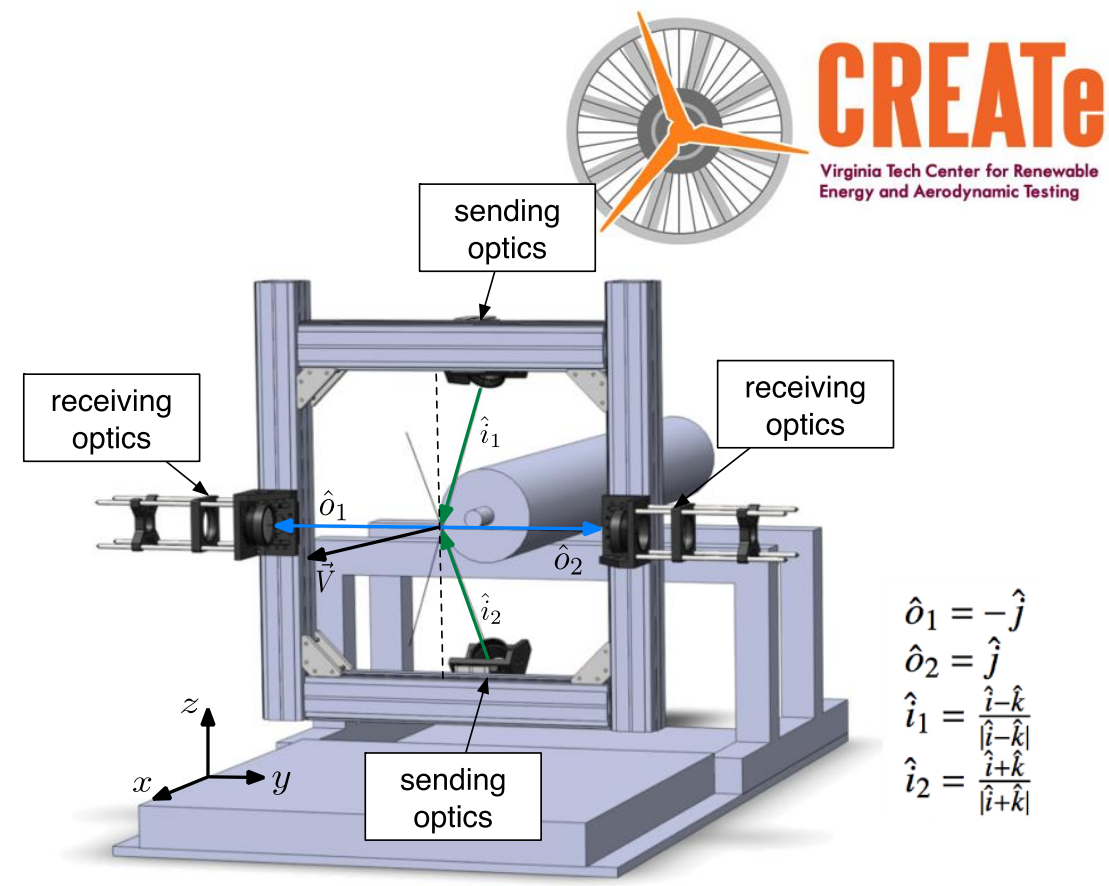

- Mean velocity, Reynolds shear stresses and velocity spectra Supersonic hot jet

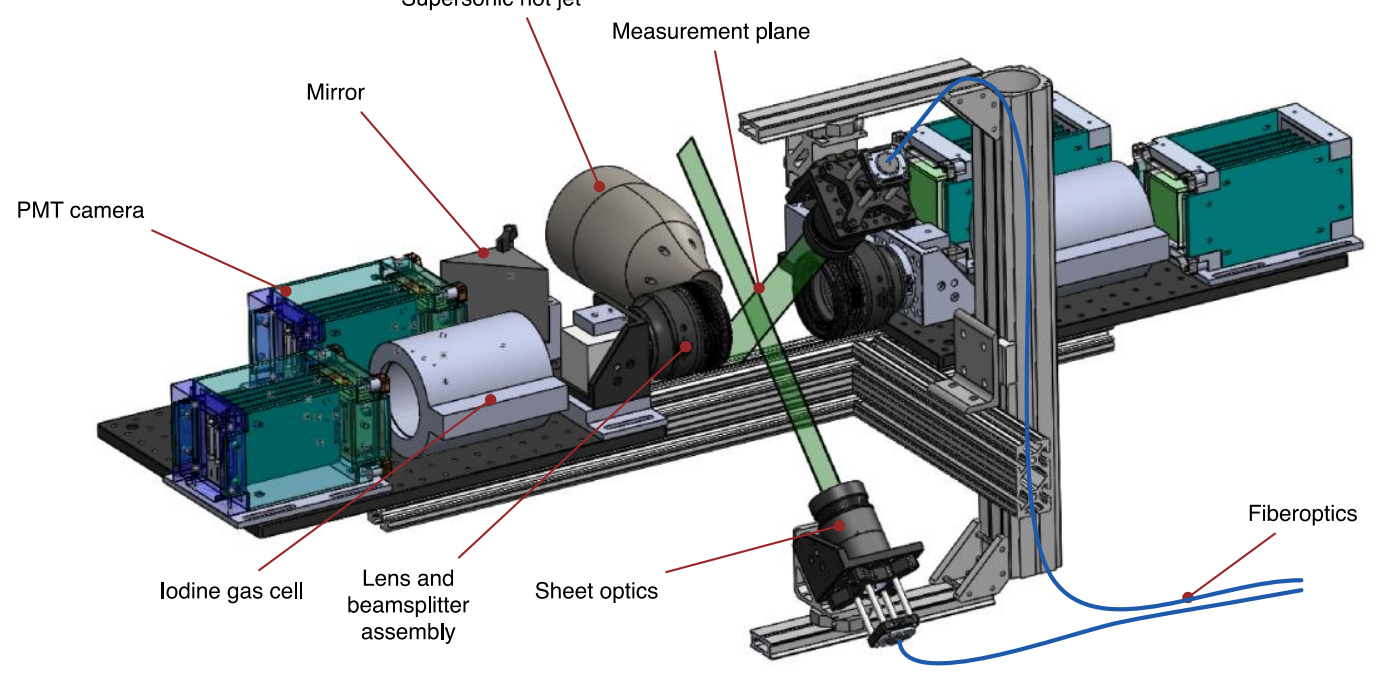

Ecker et al. (2015a,b,c)

- Multi-channel PMT cameras

- Convective velocities for heated supersonic jet

- Intermittency factor distribution

- Frequency dependent convective velocities 


\section{Key Development: PMT camera}

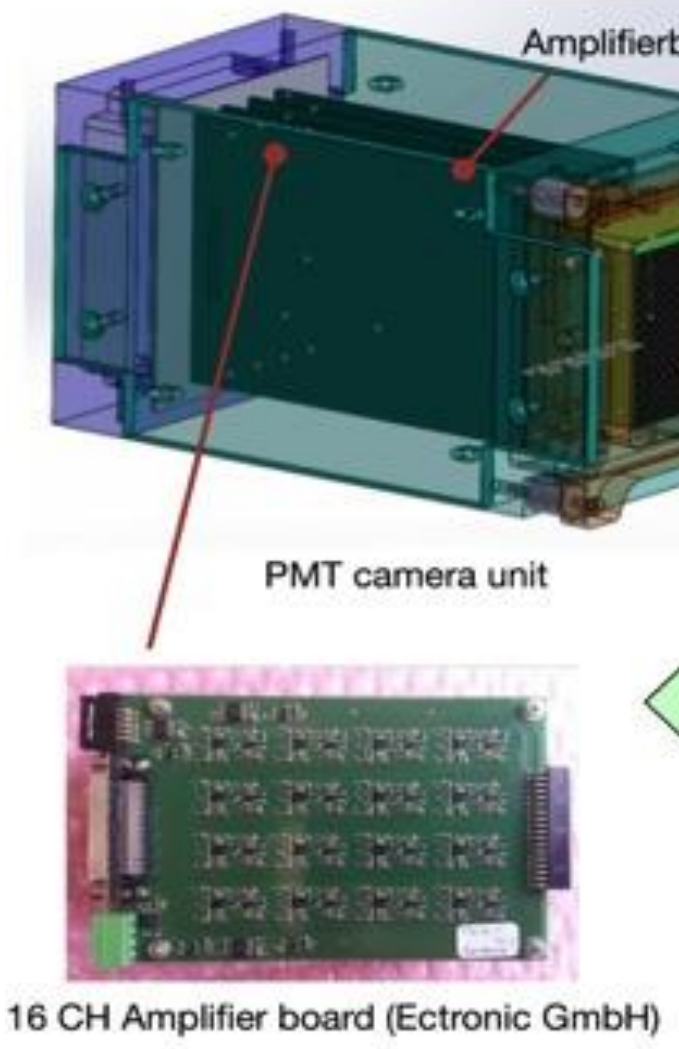

Newly developed PMT camera based on:

- Hamamatsu H8500C/H10660 64 CH PMT array

- Custom $16 \mathrm{CH}$ instrument amplifier boards

- FPGA based DAQ backend allows recording at $50 \mathrm{MHz}$ sampling rate. Preprocessing on the FPGA reduces actual streaming bandwidth to 10MS/s per channel 


\section{Time-resolved Doppler Global Velocimetry}

Doppler sensitive PMT camera

lodine cell beamsplitter

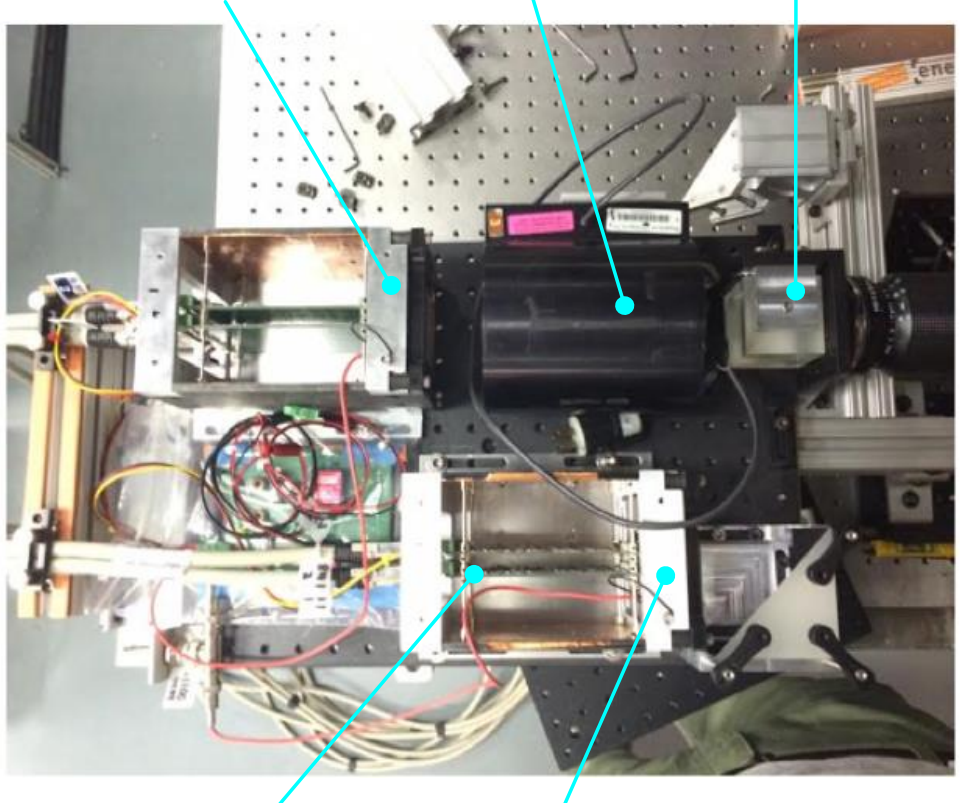

amplifier banks reference PMT camera lens

blackout cover

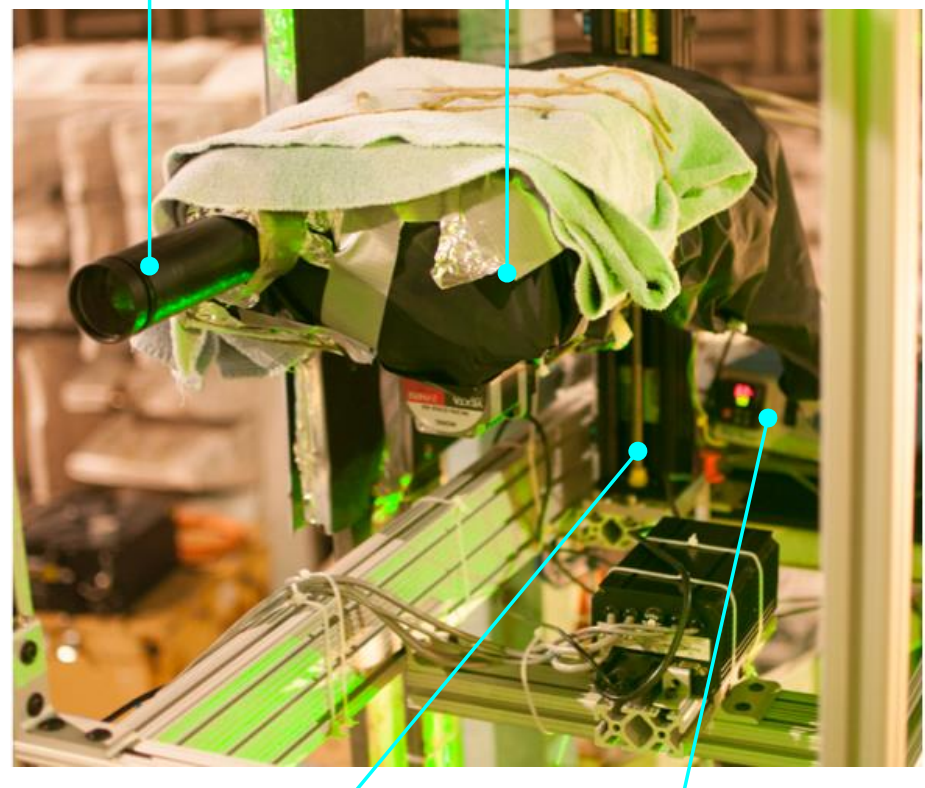

traversing unit controller for iodine cell heater

\begin{tabular}{|c|}
\hline Lens focal length \\
\hline Magnification \\
\hline Sensor area (effective) \\
\hline Measurement area \\
\hline (horizontal) spatial resolution on \\
measurement plane
\end{tabular}

24.

$200 \mathrm{~mm}$ 1.257

$24.12 \times 48.7 \mathrm{~mm}^{2}$ $30.32 \times 61.22 \mathrm{~mm}^{2}$

$7.58 \mathrm{~mm}$
- $250 \mathrm{kHz}$ flow sampling

- 3-velocity component capability

- 32-points simultaneously sampled 


\section{Facility}

Tests in Nozzle Acoustics Test Rig (NATR) and High Flow Jet Exit Rig (HFJER) at the NASA GRC Aero-Acoustics Propulsion Laboratory

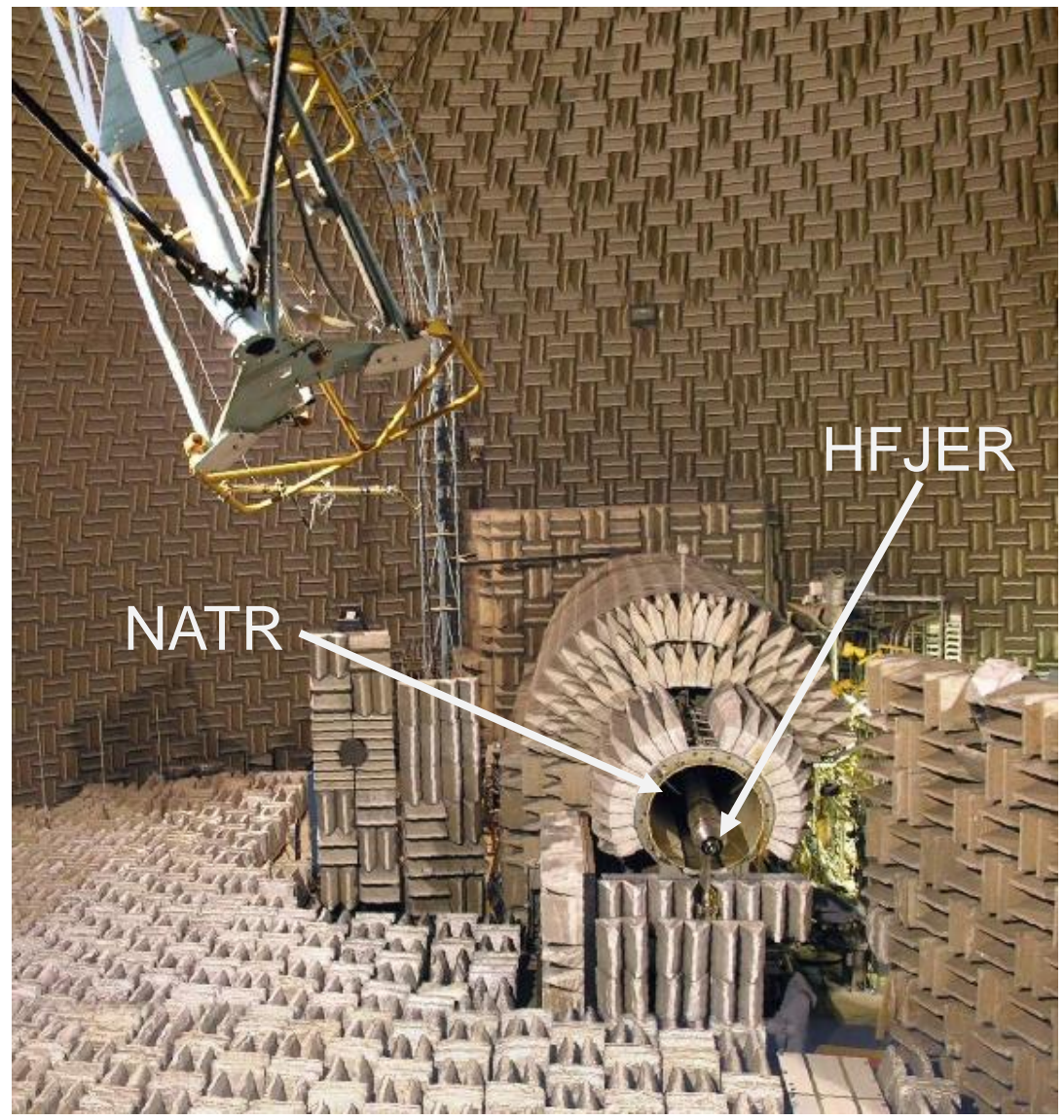

- 65 feet high, 130 feet diameter AAPL dome

- Anechoic testing environment

- Engine component R\&D

- NATR:, 53 inch diameter, freejet acoustic wind tunnel

- HFJER to mount test nozzle hardware within NATR

http://facilities.grc.nasa.gov/aapl/ 


\section{Instrument arrangement in facility}

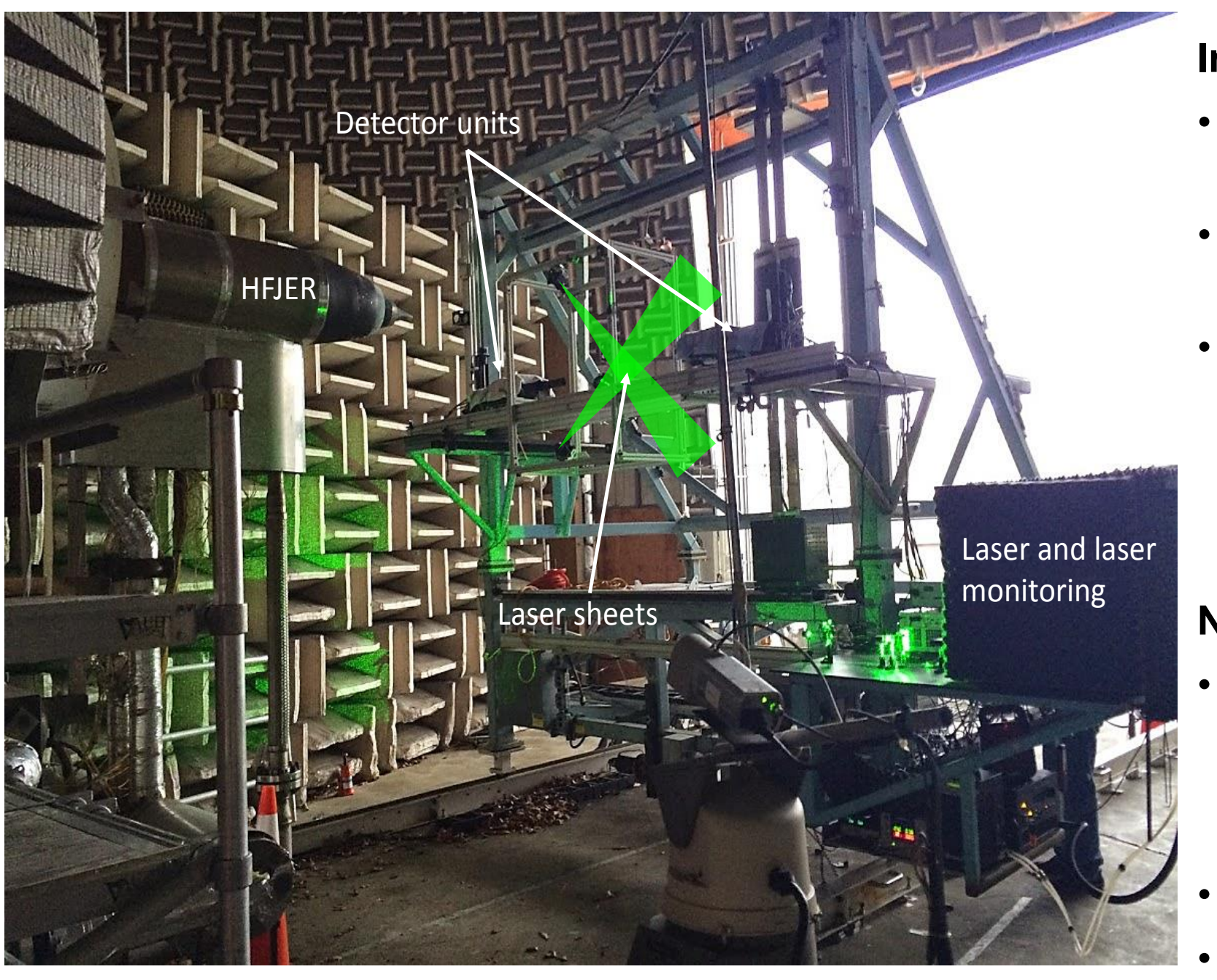

Installation:

- Used NASA facility traverse

- All optics, electronics on traverse

- Acoustic box for acoustic and thermal shielding of Laser and reference gas cell

Notes:

- Operation robust over $25^{\circ} \mathrm{C}$ temperature changes (and freezing temps)

- More seeding than PIV

- Laser frequency stabilization

Configuration instantaneous velocity uncertainty $\pm 9 \mathrm{~m} / \mathrm{s}$ 


\section{Three-stream experiments}

\begin{tabular}{l|l}
\hline Area ratio: & \\
\hline$A_{t} / A_{c}$ & 1.0 \\
\hline$A_{b} / A_{c}$ & 2.5 \\
\hline $\begin{array}{l}\text { Nozzle pressure ratio: } \\
\text { NPR: core and bypass }\end{array}$ & 1.8 \\
\hline NPR: tertiary & 1.4 \\
\hline Nozzle temperature ratio: & \\
\hline NTR: core & 3.0 \\
\hline
\end{tabular}

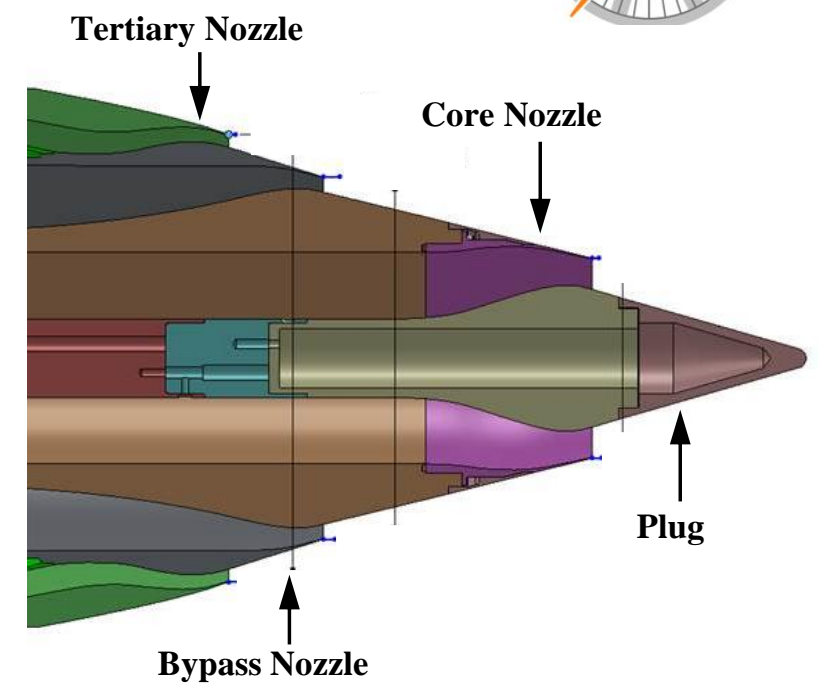

The axisymmetric-nozzle system used in the three-stream experiments.

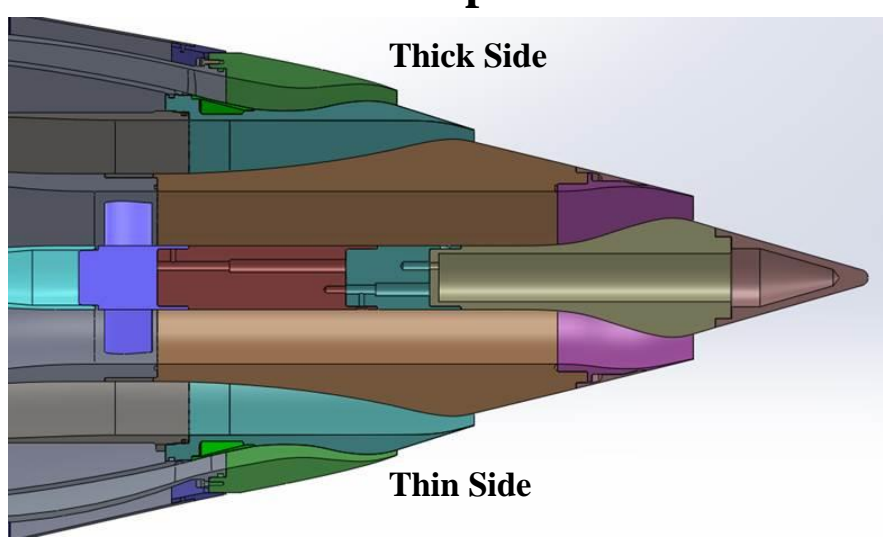

The offset nozzle system used in the three-stream experiments. 


\section{Velocity results: Validation}

Comparison PIV data: Henderson and Wernet (2016)

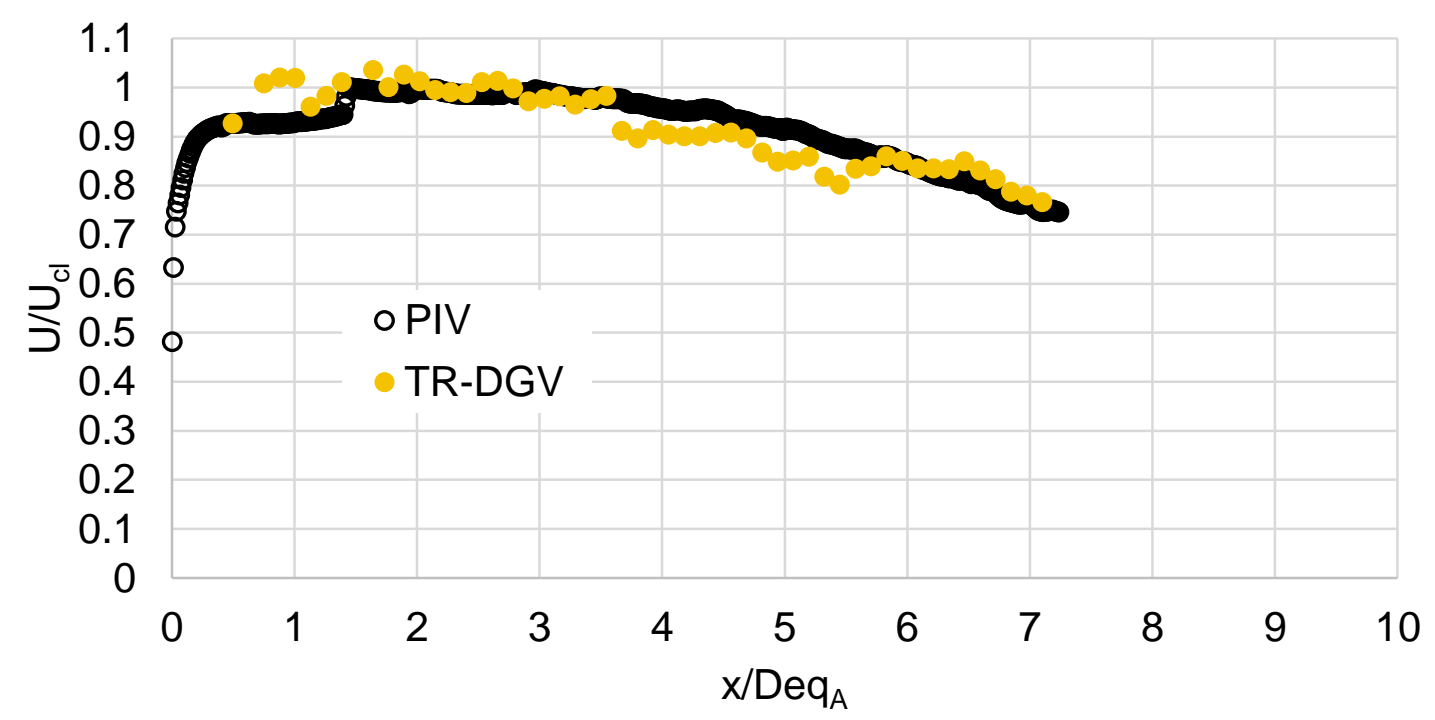

- Laser frequency fluctuations

- Mean velocity comparison reasonable

- Turbulence results show some differences

- Instrument variance

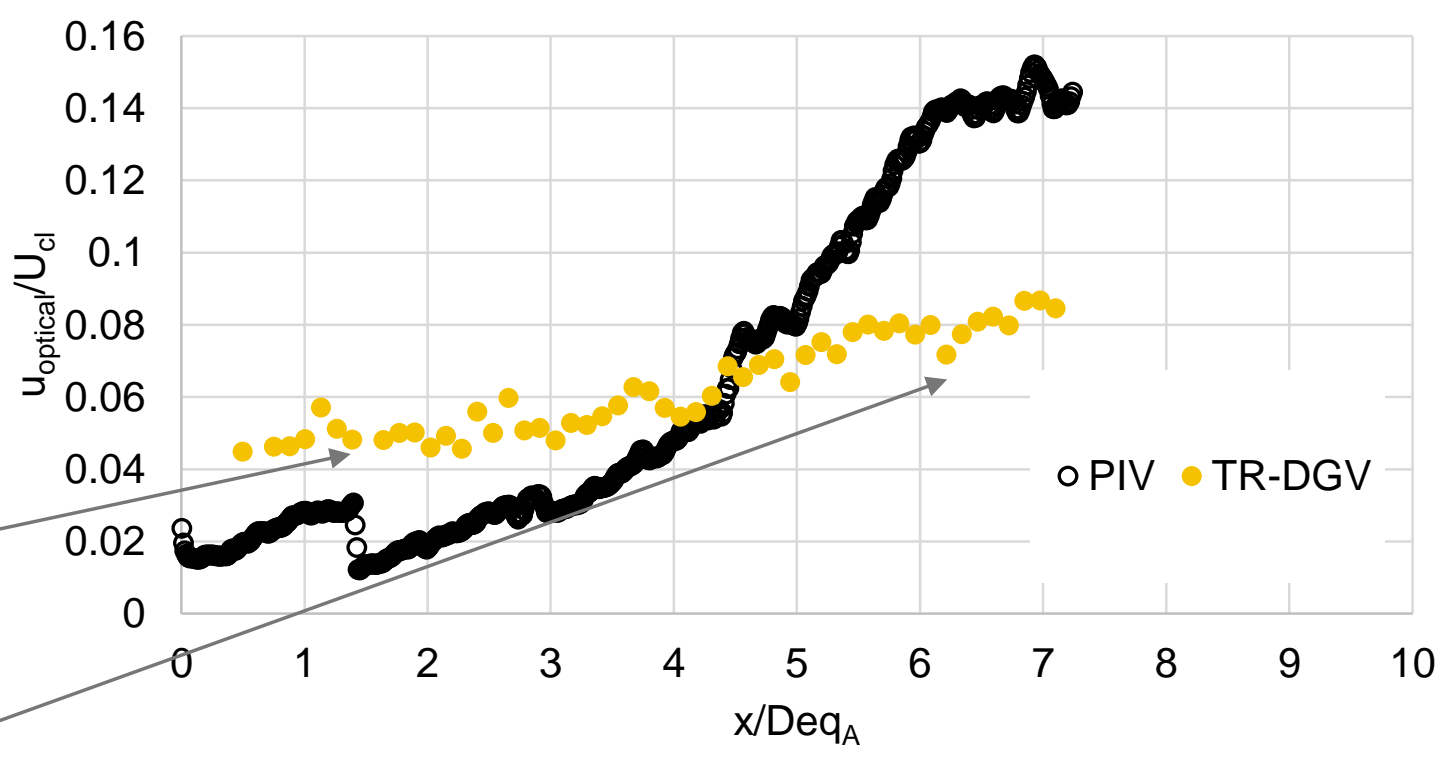




\section{Velocity spectra: Axisymmetric nozzle on centerline,}

Stream-wise development of velocity spectra

- Consistent increase at all frequencies

- Spectral estimator important

- Drop-out

- Spectral variance

- Consistent instrumentation noise floor

- Spectral uncertainties still being quantified

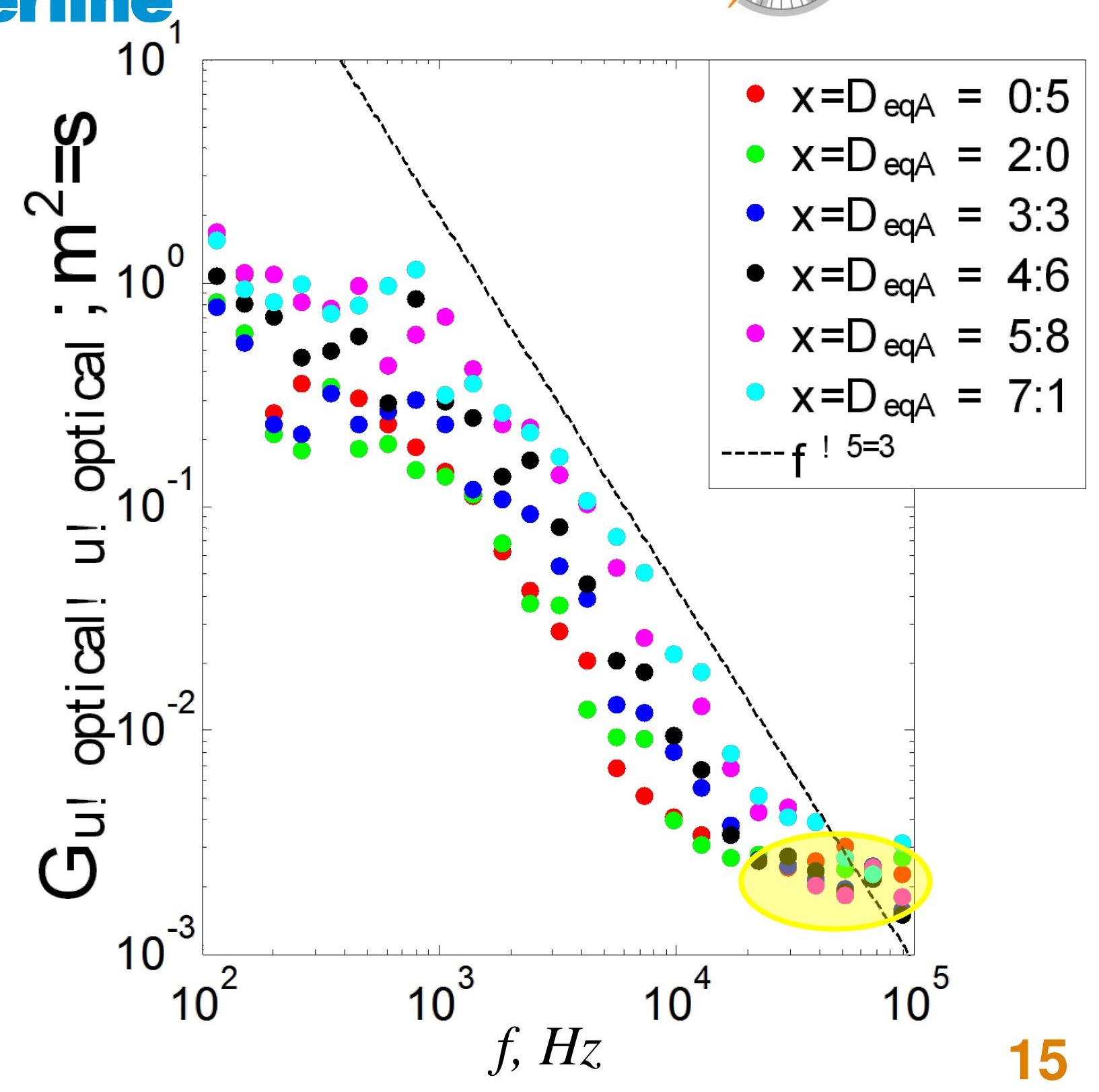




\section{Convection velocity: Axisymmetric}

Convection velocity from time/space correlation across stream-wise-spaced sensors (e.g., Ecker et

al. AIAA J. 2015, $\pm 6.5 \%$ RMS uncertainties)

2

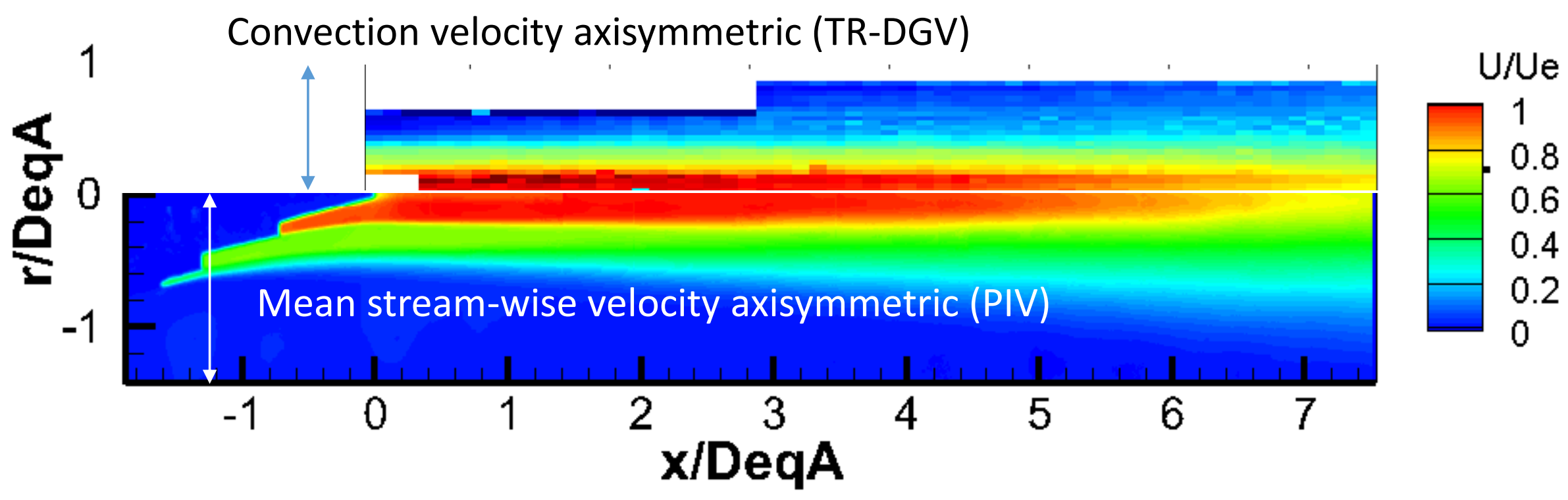

Comparison of convection and mean velocity 


\section{Convection velocity: Axisymmetric vs Offset}

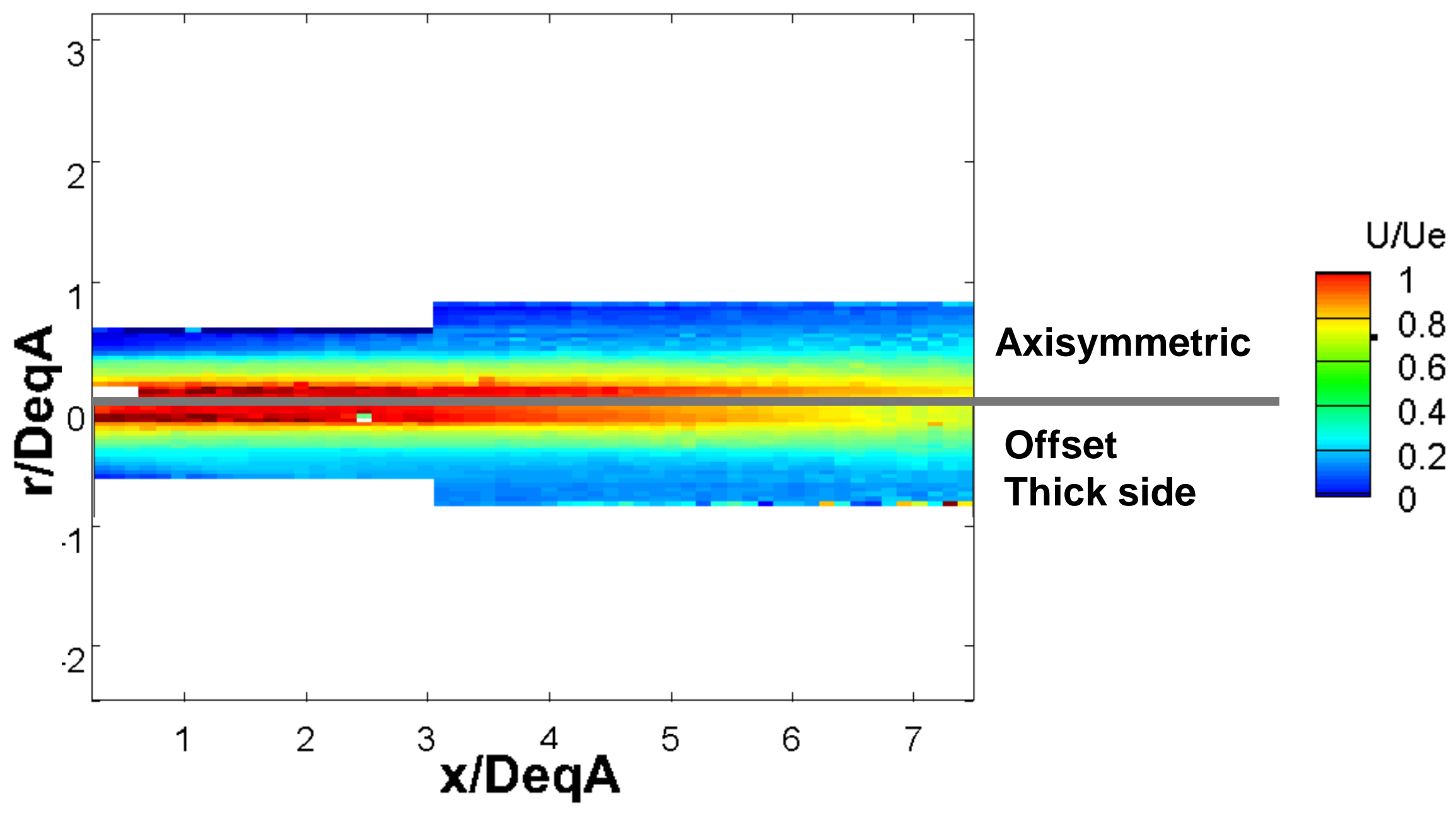

Offset clearly thickened for $x / \operatorname{Deq} A<3$

Diminishes as flow develops 


\section{Convection velocity}

Axisymmetric configuration

\section{Development of profiles} throughout this region
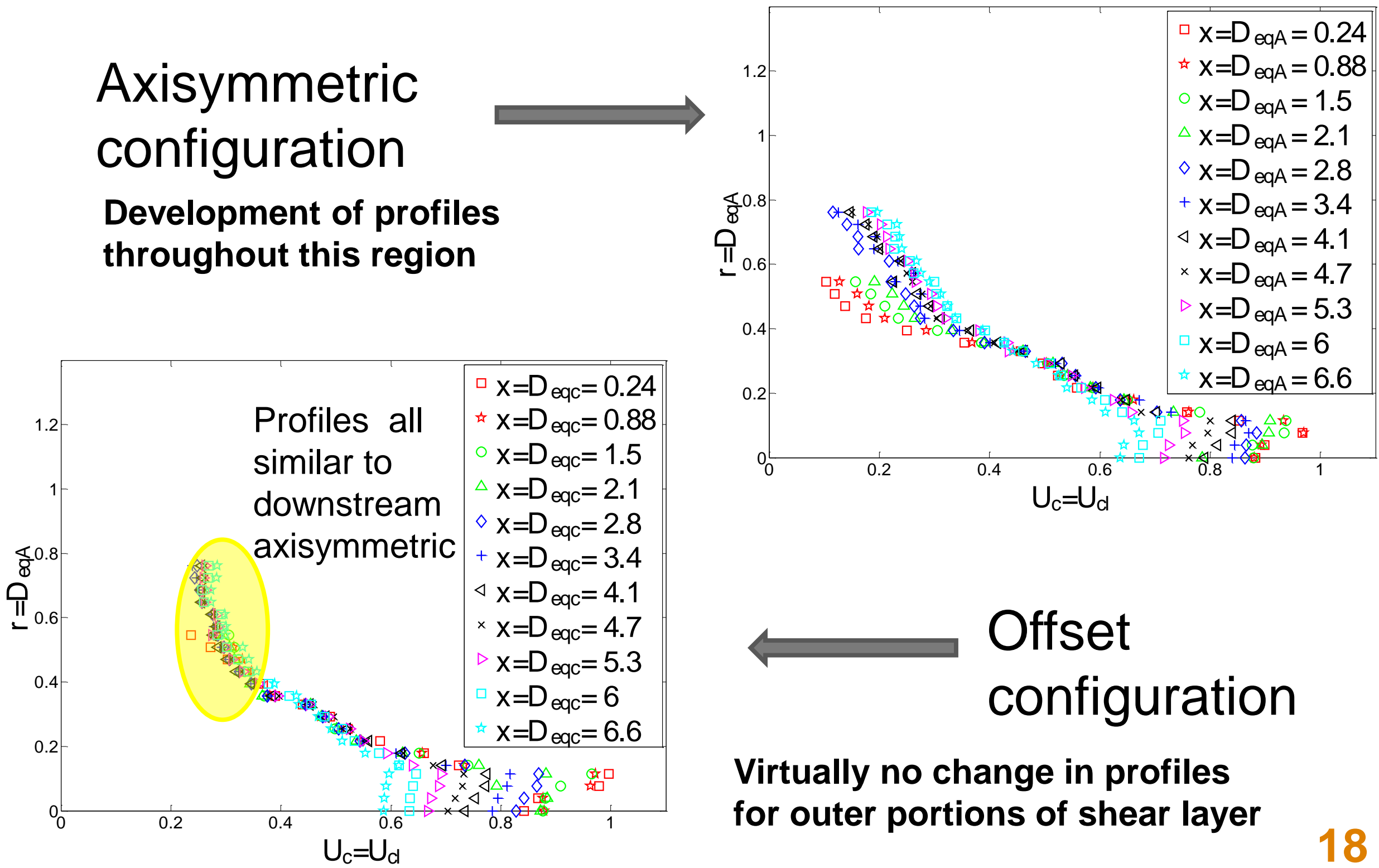

Virtually no change in profiles for outer portions of shear layer 


\section{Regarding TR-DGV}

- Discussed more instrumentation aspects in AIAA-2016-0029

- Laser frequency fluctuations

- On-going work for signal processing

- Current results first for scaled-up arrangement

- $250 \mathrm{kHz}$ velocimetry

- Shows strength of method for time-resolved data

- Work confirms complementary role for TR-DGV in conjunction with PIV
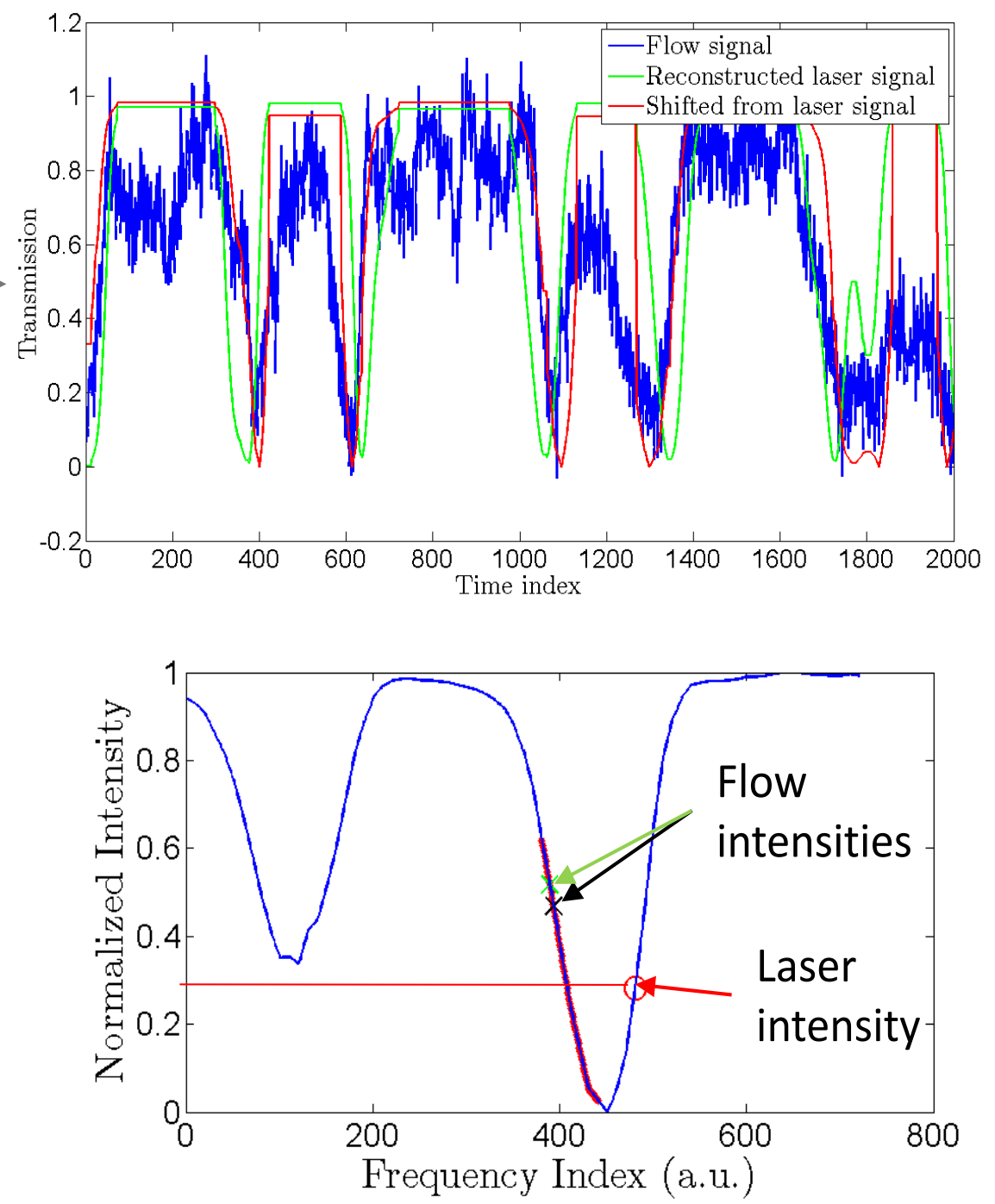


\section{Conclusions and next steps}

- Measurements using time-resolved velocimetry in 3-stream jet

- Good agreement of mean velocity data with PIV comparison data

- Turbulent intensity problematic due to noise influence, signal estimation

- Used time-resolved data to begin analysis of statistical turbulence characteristics

- Next steps

- Continue refinement of velocity estimation

- Use spectral and correlation data to update source models for three-stream jet predictions

- Analyze physics using both PIV and TR-DGV insights

- Process more data: two more configurations, one additional condition 


\section{Questions ?}




\section{Background}

\section{Review 3-stream work}

\section{NASA results previously reported}

\section{Turbulence information in 3-stream jet noise predictions}

Henderson, Brenda. "Aeroacoustics of three-stream jets." $18^{\text {th }}$ AIAA/CEAS Aeroacoustics Conference, Colorado Springs, CO, 4-6 June, 2012, AIAA Paper 2012- 2159.

Papamoschou, D., Johnson, A. D., \& Phong, V. Aeroacoustics of Three-Stream High-Speed Jets from Coaxial and Asymmetric Nozzles. Journal of Propulsion and Power, 30(4), 2014, 1055-1069.

Simmons, S. P., Henderson, B., \& Khavaran, A. "Flow Field and Acoustic Predictions for Three-Stream Jets," $50^{\text {th }}$ AIAA/ASME/SAE/ASEE Joint Propulsion Conf., Cleveland, OH, 2014.

Henderson B. S., Bridges J. and Wernet M.P., "Jet Noise Reduction Potential From Emerging Variable Cycle Technologies," 48th Joint Propulsion Conference and Exhibit cosponsored by the AIAA, ASME, SAE, and ASE Atlanta, Georgia, July 30August 1, 2012.

${ }^{19}$ Khavaran A. and Bridges J., "Jet Noise Scaling in Dual Stream Nozzles", AIAA2010-3968, 2010. 


\section{Convection velocity: Offset}

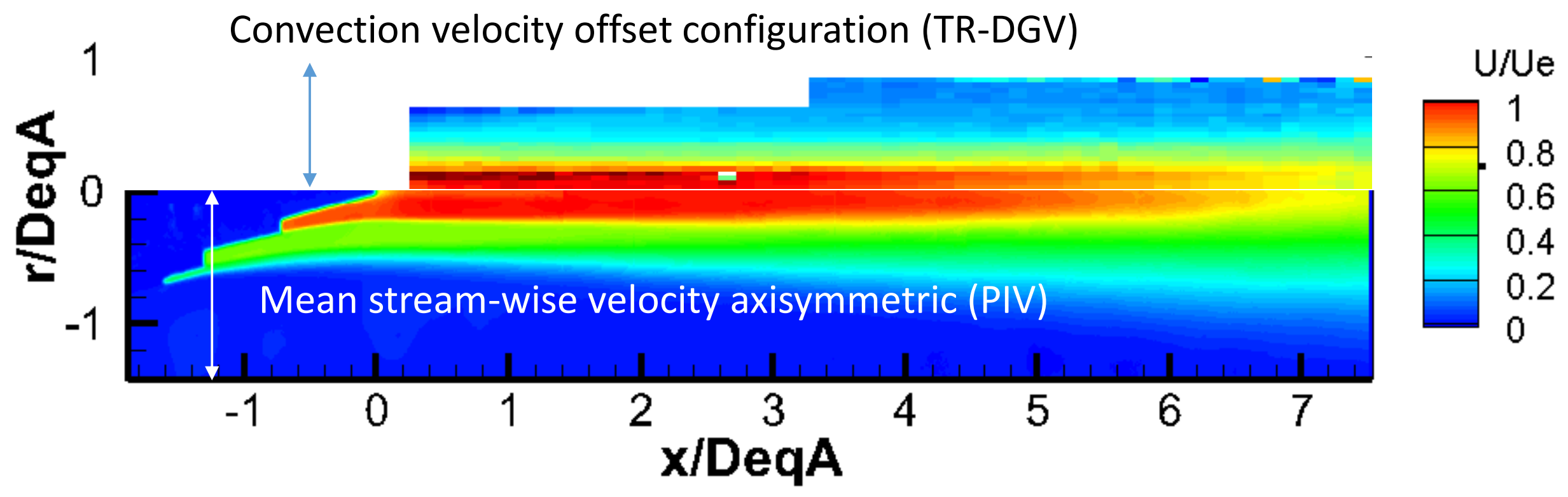

Offset configuration convection velocity compared to mean velocity of axisymmetric case (reference) 


\section{TR-DGV geometry}

- 45 deg. arrangement

- Uncertainty (inst) $9.2 / 6.5 /$ $6.2 \mathrm{~m} / \mathrm{s}$

- Uncertainty (mean) 1.5/1.5 $/ 1.5 \mathrm{~m} / \mathrm{s}$

$$
u_{\text {optical }}=-\frac{\sqrt{2}}{2} u+\frac{\sqrt{2}}{2} v+w
$$

$$
\begin{gathered}
\delta u_{i}=\frac{c}{f_{0}} \sqrt{\left(\frac{d f}{d T}\right)_{i}^{2}\left(\delta T_{i}\right)^{2}+T_{i}^{2}\left[\delta\left(\frac{d f}{d T}\right)_{i}\right]^{2}+\frac{f_{i}^{2}}{f_{0}^{2}}\left\{\left(\frac{d f}{d T}\right)_{0}^{2}\left(\delta T_{0}\right)^{2}+T_{0}^{2}\left[\delta\left(\frac{d f}{d T}\right)_{0}\right]^{2}\right\}} \\
\Delta D=\left(\begin{array}{l}
\delta \boldsymbol{u}_{\boldsymbol{x}} \\
\delta \boldsymbol{u}_{\boldsymbol{y}} \\
\delta \boldsymbol{u}_{\boldsymbol{z}}
\end{array}\right)=\sqrt{\left(\left[\begin{array}{lll}
R_{11} & R_{12} & R_{13} \\
R_{21} & R_{22} & R_{23} \\
R_{31} & R_{32} & R_{33}
\end{array}\right]\right)^{2}\left(\begin{array}{l}
\delta u_{1} \\
\delta u_{2} \\
\delta u_{3}
\end{array}\right)^{2}+\left(\left[\begin{array}{lll}
\delta R_{11} & \delta R_{12} & \delta R_{13} \\
\delta R_{21} & \delta R_{22} & \delta R_{23} \\
\delta R_{31} & \delta R_{32} & \delta R_{33}
\end{array}\right]\right)^{u_{1}}\left(\begin{array}{l}
u_{2} \\
u_{3}
\end{array}\right)^{2}}
\end{gathered}
$$

